\title{
Influence of Geometrical Parameters on the Flexural Rigidity of the LHC Dipole Cold Mass Assembly
}

\author{
M. Bajkó, R. Chamizo, and A. Pardons
}

\begin{abstract}
In order to predict the mechanical behavior of the LHC dipole cold mass in situations such as handling, transport and cool down, a number of important structural parameters are required. The dipole's flexural rigidity determines entirely the mechanical elastic behavior of the cold mass. Therefore, models of a bent cold mass were created to calculate its rigidity. This paper presents a simplified parametric finite element model, created to study the deflection of the cold mass in different situations and supporting conditions. The sensitivity of the models to the supporting conditions is computed. To provide the finite element and the analytical models with input, the deflection of the cold mass under discrete loads in normal condition and then 90-degrees rotated were measured with a laser tracker. By comparing models with measurements, the vertical and transversal rigidity of the cold mass assembly are determined. Additionally, the paper reports on the plastic behavior of the cold mass assembly in the range of the deformations that are needed to correct cold masses that result, after final welding of the outer skin, with unacceptable sagitta.
\end{abstract}

Index Terms-Alignment, flexural rigidity, geometry, LHC dipole cold mass assembly.

\section{INTRODUCTION}

$\mathbf{T}$ HE MECHANICAL behavior of the LHC dipole cold mass $(\mathrm{CM})$ in different conditions has to be known for many reasons. The handling of the $\mathrm{CM}$ should be controlled in order to assure that the correct geometry of the cold mass be conserved until its installation in the tunnel. The large beam size at the injection, put demands on the shape and the positioning tolerances of the axes of the dipole cold bore tubes. There are also strict requirements dictated by the mechanical interconnections between adjacent magnets. In order to respect the alignment requirements in the tunnel, tight tolerances are imposed during the final assembling steps of the dipole CM, as well as during cryostating and fiducialization. The total tolerance "budget" is shared between these operations and there is no room for extra errors coming from noncontrolled handling or any other harmful operations. However, following the life of the dipoles from assembly to installation in the tunnel, several transport and handling operations are foreseen. For these operations it is important to define a maximum permitted deformation. Once a CM is installed and aligned in the tunnel, it will be cycled between room temperature and $1.9 \mathrm{~K}$ several times during the LHC lifetime. Mechanical models are needed to describe the behavior of the dipole CM in these different situations, allowing to define the conditions for transport,

Manuscript received September 24, 2001.

The authors are with CERN, Geneva, $\mathrm{CH}$ (e-mail: \{Marta.Bajko; Rocio.Chamizo; Ans.Pardons\} @cern.ch).

Publisher Item Identifier S 1051-8223(02)04272-0.

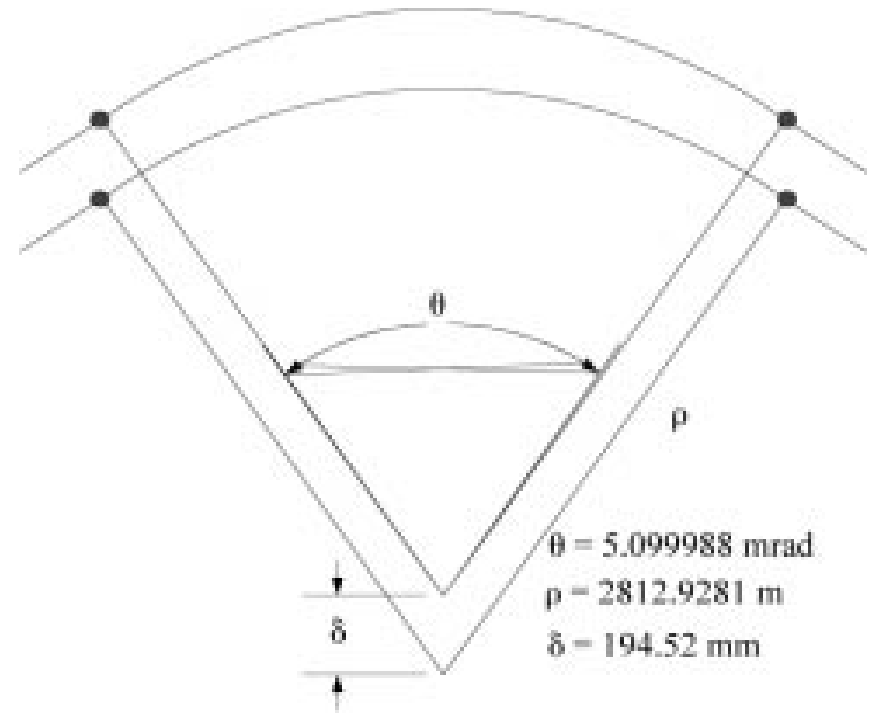

Fig. 1. Theoretical geometry of the dipole $\mathrm{CM}$ at room temperature.

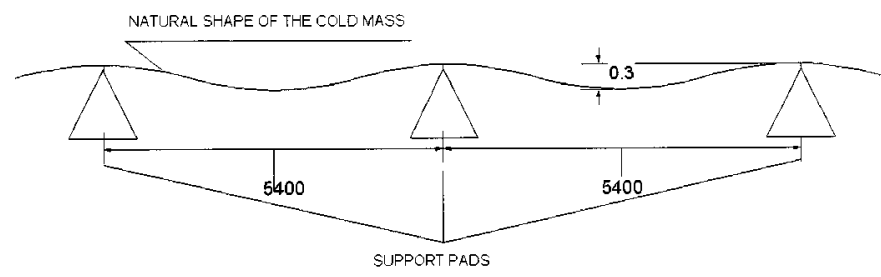

Fig. 2. The natural shape of the dipole cold mass in the vertical plane.

handling and to predict the shape of the CM after thermal cycling. In this paper it is given an estimation of the flexural rigidity of the dipole $\mathrm{CM}$, which can then be input to a 3-D mechanical model describing the geometry variation during transport and thermal cycle. In addition a "recipe" is provided for the corrective action to be performed on the CM geometry, if necessary, in a relatively early stage of the CM assembly.

\section{ShORT DESCRIPTION OF THE DIPOLE COLD MASS}

\section{A. The Geometry of the Dipole Cold Mass}

The shape of the two beam channels is identical. The centers of curvature of the apertures are $194 \mathrm{~mm}$ apart at nominal working temperature $(1.9 \mathrm{~K})$, which correspond to $194.52 \mathrm{~mm}$ at room temperature. In the vertical plane, the ideal shape of each cold bore tube (CBT) axis is a straight line, but in reality the CM has a finite bending stiffness, and is supported only in three points [1]. 


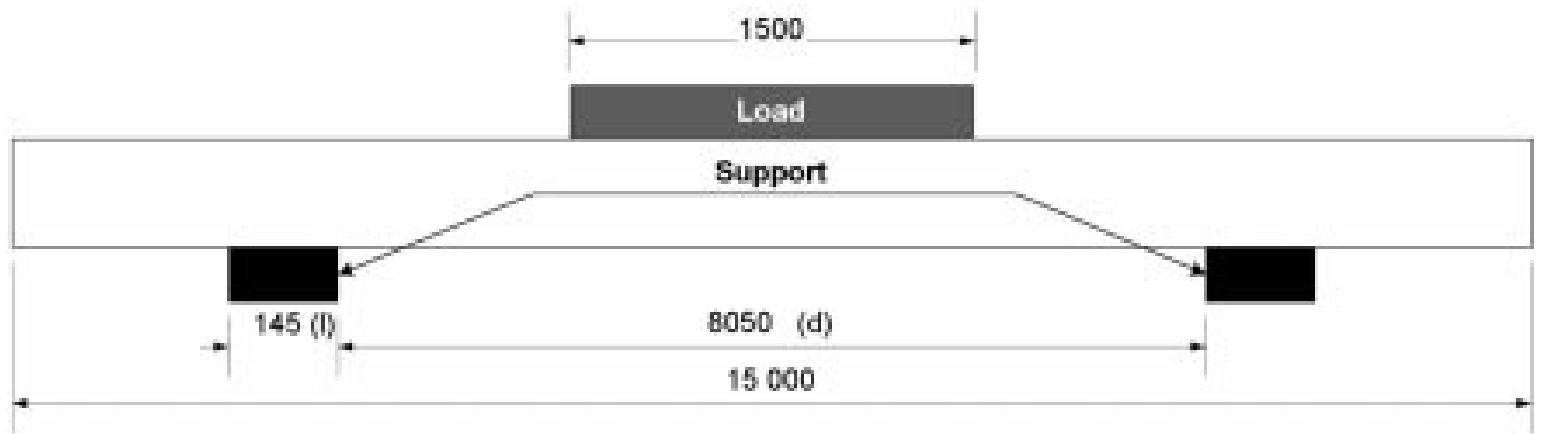

Fig. 3. The experimental set-up nr. 1.

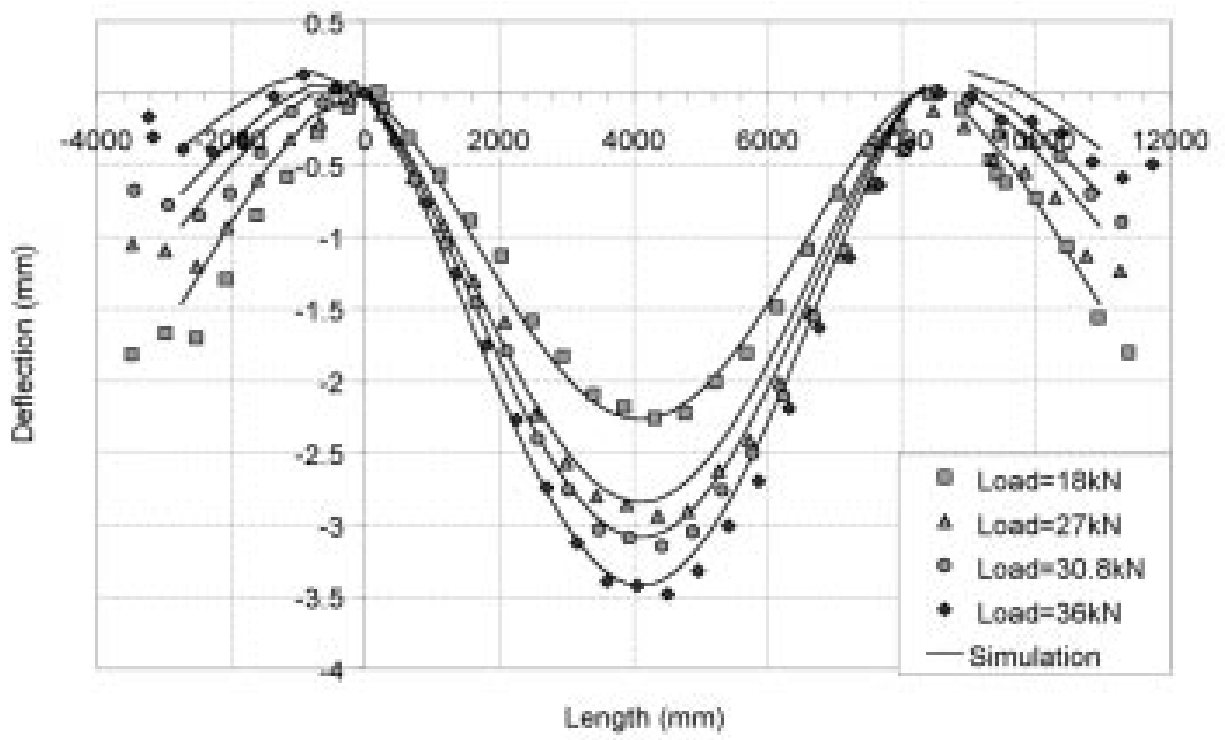

Fig. 4. The axis of one of the CBT of the dipole CM under 4 different loads. Absolute deflection in the vertical plane (points). Simulated results from FE model (continuous line).

\section{B. The Main Components of the Dipole Cold Mass}

The active part is made of:

1) Two dipole coils, each consisting of an upper and a lower pole. Each pole combines two superconducting windings, called the inner and outer layer, which are electrically connected.

2) A common nonmagnetic, force-retaining laminated structure made up of austenitic steel collars. This structure confines and pre-stresses the coils, strictly maintaining their geometry in presence of the very high electromagnetic forces occurring during magnet testing and operation. These common collars assure the distance $\delta$ of $194.52 \mathrm{~mm}$ between the two dipole coils.

3) A laminated iron yoke split into two parts in the vertical symmetry plane, providing the return path for the magnetic flux and conferring mechanical rigidity to the whole structure.

4) Two laminated iron inserts placed across the vertical symmetry plane.

5) An austenitic steel shrinking cylinder, made of two welded half-cylinders, surrounds the yoke. This cylinder gives the CM assembly the stiffness needed to contain the electromagnetic forces, it provides the inertia necessary to maintain the geometry of the CM and it constitutes, with the end covers, the superfluid helium vessel.

6) Two austenitic steel end plates, closing the active part at both extremities, and absorbing the longitudinal electromagnetic forces. Items 1 and 2 together with the related cold bore tubes constitute a sub-assembly that will be hereafter referred to as the "collared coils" [2].

Fig. 6 shows items $1-5$ in a cold mass cross-section.

The whole assembly has a weight of $275 \mathrm{kN}$ and is, in nominal conditions, on 3 supports. The support pads are $5.4 \mathrm{~m}$ apart. In nominal supporting conditions, the dipole cold mass is deflected in the vertical plane with a sagitta of $0.3 \mathrm{~mm}$.

\section{Method to Determine the Flexural Rigidity of the DIPOLE COLD MASS}

In order to determine the flexural rigidity (bending stiffness) of the dipole cold mass a number of measurements were performed on different cold masses, in different supporting and loading conditions. These loads and supporting conditions were 


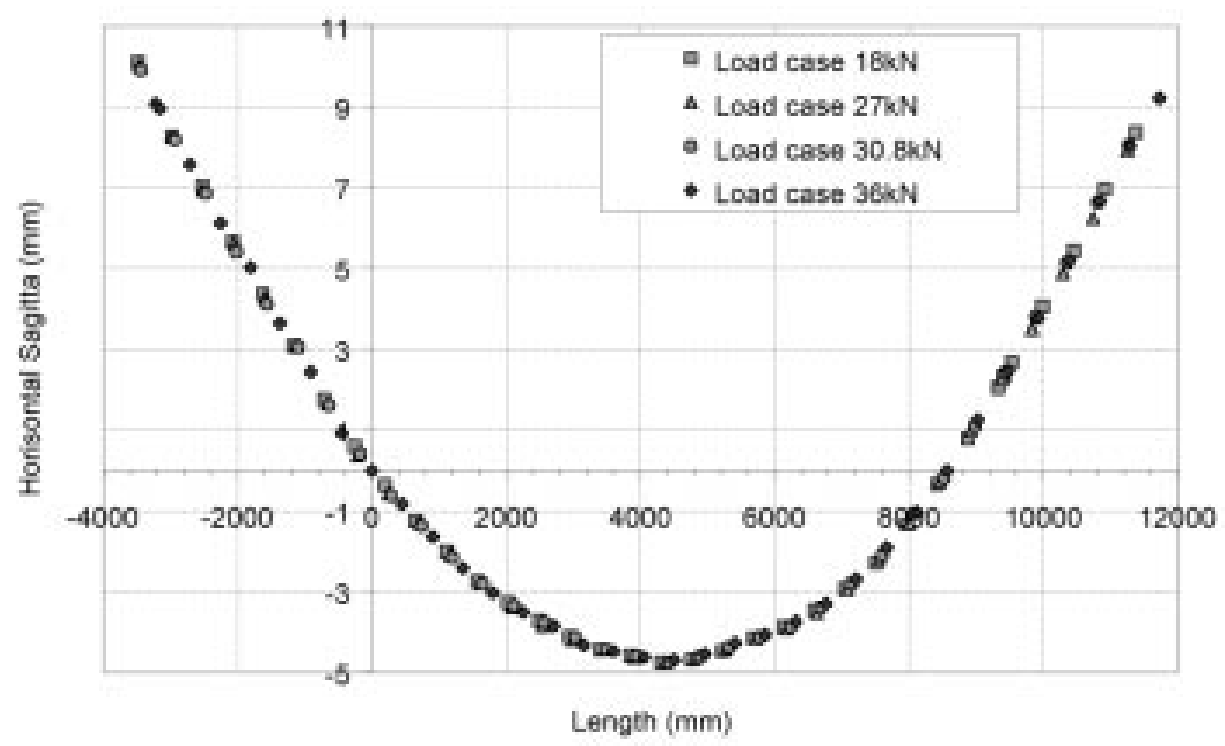

Fig. 5. The shape of $\mathrm{CM}$ in the transversal plane when the vertical plane is charged with different loads.

introduced into a finite element model (FEM) of the cold mass. By adapting the bending stiffness of the model in order to fit the calculated deflection with the measurements allowed, after a few iterations, to determine the magnet stiffness.

\section{A. Experimental Set-Up}

In order to avoid hyperstatic (statically indeterminate) cases and to minimize the number of factors influencing the sensitivity of the FE-model, the dipole cold mass was placed on two supports and charged in the middle by various distributed loads. Since an existing bench for the final CM assembly was used for these measurements, the distance between the two supports was fixed to approximately $8 \mathrm{~m}$. The cold mass was placed symmetrically on the bench with respect to the supports. This simple experimental set-up avoids the need of a rigid structure around the cold mass for the loading. Loads are constituted by blocks placed on the top of the cold mass, half way between the two supports, as shown in Fig. 3.

For each loading case, the axis of a CBT was measured over its whole length. The same measurement was repeated in order to check for residual deformations, i.e., the start of a plastic behavior. A cold mass CBT was measured under and after four different loads. The combination of the blocks permitted the following four cases: CM charged with $18 \mathrm{kN}$, with $27 \mathrm{kN}$, with $30.8 \mathrm{kN}$ and with $36 \mathrm{kN}$. The deformations of the CM in the vertical plane under these four loading cases are shown in Fig. 4. As a reference, the natural sagitta with this support configuration is $0.6 \mathrm{~mm}$.

The deformations in the transversal plane are also recorded to study a possible cross-talk between the loaded (vertical) and transversal plane, i.e., the influence of the deformation in the vertical plane on the deformation in the transversal plane and vice versa. In the range of the applied loads, no cross-talk has been observed. The shape of the CM in the transversal plane in the different cases is shown in Fig. 5.

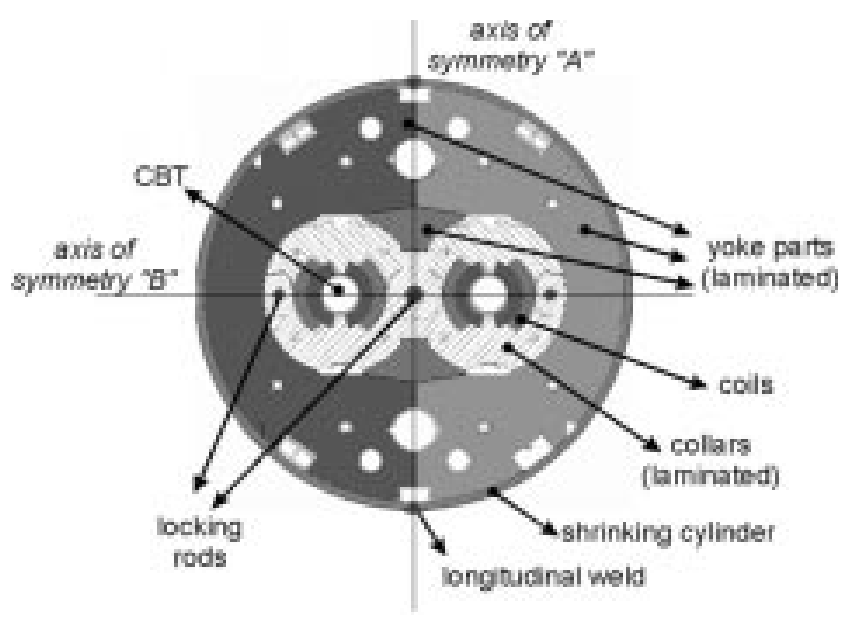

Fig. 6. The cross section of the cold mass.

Fig. 6 shows the CM cross-section and the main components contributing to the magnet's stiffness: the shrinking cylinder with longitudinal welds, the coils, the half-yokes, the collars with locking rods and the cold bore tubes. From this cross-section, it is clear that the transversal stiffness (bending around axis "A") will be considerably different from the vertical stiffness (bending around axis "B"). The position of the coils, locking rods and cold bore tubes with respect to axes A and B suggests that the horizontal rigidity is higher than the vertical rigidity.

In order to determine the transversal stiffness, the magnet was turned 90 degrees from its nominal working position. Then the magnet, with the sagitta facing down, was loaded with discrete forces up to $130 \mathrm{kN}$. This test also allows to determine the regime of plastic deformation of the cold mass. Three supports are placed along the longitudinal axes. Two shims were placed on the supports to follow the initial curvature of the CM. On the two extremities of the CM, a local load was applied using the rigid structure of the welding press (see Fig. 7). 


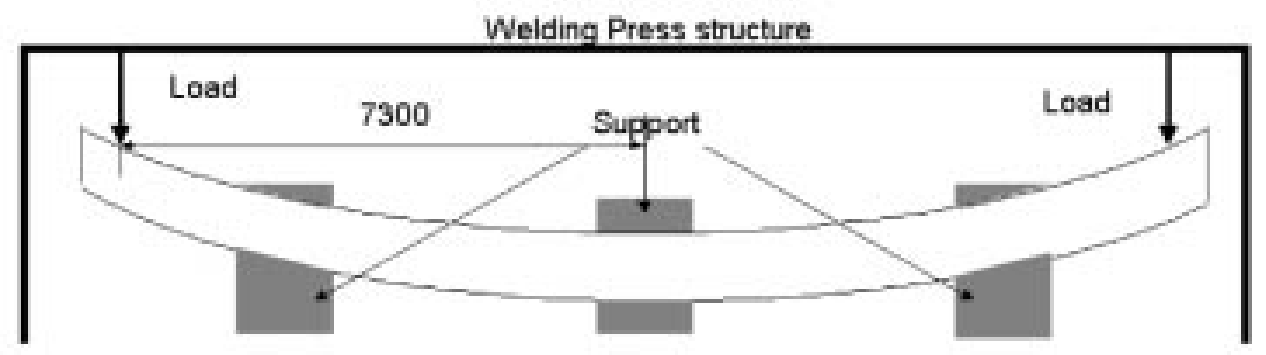

Fig. 7. The experimental setup nr. 2.

\section{B. The Modeling}

In order to determine the flexural rigidity, a simple 2-D finite element model (FEM) is made. The cold mass is represented by 2-D beam elements and is modeled as a cylinder with the geometrical and mechanical characteristics of the shrinking cylinder, and a weight of $275 \mathrm{kN}$. The geometrical and mechanical characteristics of the cylinder are listed in the Table I.

A factor " $f$," varying between 1 and 1.5 , is introduced to scale the inertia " $I$ " of the cylinder to the inertia of the whole CM assembly, meaning $f=I_{C M} / I_{c y l}$. The distance between the supports (" $d ")$ and the length of the contact surface between each support and the cold mass ("l") are important parameters of the mechanical model. These two factors have a significant influence on the calculated deflection, comparable to that obtained by the relative variation of the factor " $f$." Since the relation between the maximum deflection of the cold mass and the contact length of the support can not be expressed analytically, a statistical relation is given. Several cases are considered varying the parameters $f, d$, and $l$. The parameters and their range of variation are listed in Table II.

Combining the results from several models, all loaded with a force of $36 \mathrm{kN}$ but with different values for the factor $f$ and the distance $d$, the maximum deflection $y_{\max }$, can be expressed as follows:

$$
y_{\max }(f, d)=\alpha \cdot f^{-1}+\beta \cdot d^{3}+\text { const }_{1}
$$

where $\alpha=-3.59, \beta=-1.5510^{-11}$ and const $_{1}=8.86$ result from least square based fitting. In a similar way the maximum deflection can be formulated as a function of the factor $f$ and of the contact length $l$ :

$$
y_{\max }(f, l)=\chi \cdot f^{-1}+\delta \cdot l^{2}+\varepsilon \cdot l+\text { const }_{2}
$$

with $\chi=-3.07, \delta=-5.210^{-5}, \varepsilon=0.018{\text { and } \text { const }_{2}=}^{-5}$ -2.01 , where " $y_{\max }$," " $l$ " and " $d$ " are expressed in millimeters.

The sensitivity of the model to the variation of the parameters $f, d, l$ is given by the partial derivation of the functions:

$$
\begin{array}{llrl}
\frac{\partial y_{\max }(f, d)}{\partial f}=-\alpha \cdot f^{-2} & \text { or } & \frac{\partial y_{\max }(f, l)}{\partial f}=-\chi \cdot f^{-2} \\
\frac{\partial y_{\max }(f, d)}{\partial d}=3 \beta \cdot d^{2} & \frac{\partial y_{\max }(f, l)}{\partial l}=2 \delta \cdot l+\varepsilon
\end{array}
$$

This means that a variation of $0.1 \mathrm{~mm}$ of the maximum deflection can be caused by an error of $4 \mathrm{~mm}$ on the defined contact
TABLE I

CHARACTERISTICS OF THE CYLINDER

\begin{tabular}{lll}
\hline \multicolumn{1}{c}{ Parameter } & \multicolumn{1}{c}{ value } \\
\hline Outer diameter of the cylinder & De & $0.571 \mathrm{~m}$ \\
Inner diameter of the cylinder & Di & $0.550 \mathrm{~m}$ \\
Overall length of the cold mass & $\mathrm{L}$ & $15.160 \mathrm{~m}$ \\
Young modulus & $\mathrm{E}$ & $195 \times 10 \mathrm{~Pa}$ \\
\hline
\end{tabular}

TABLE II

INPUT PARAMETERS OF THE MODEL AND THEIR RANGE OF VARIATION

\begin{tabular}{lll}
\hline \multicolumn{1}{c}{ Parameter } & Rarnge of variation \\
\hline Inertia factor & f & $1.1-1.3$ \\
Distance between the supports & d & $8050-8200 \mathrm{~m}$ \\
Contact length & I & $40-140 \mathrm{~m}$ \\
\hline
\end{tabular}

length, $30 \mathrm{~mm}$ error on the given distance between the supports or by an error of $3.3 \%-3.8 \%$ on the factor $f$, related to the estimation of the cold mass inertia and, consequently, the magnet stiffness.

A FE model with contact elements confirmed that, under the four load cases considered (Fig. 4) and for a support length in the range 0.04 to $0.14 \mathrm{~m}$, contact occurs over the entire support length. This means that the factor " $l$ " from Table II can be determined by measuring the length of the actual supports. Consequently, both " $l$ " and " $d$ " (the distance between the supports) can be known with an accuracy of $\pm 1 \mathrm{~mm}$, leaving only the precision of the measurement device as a limit to determine accurately " $f$ " or the magnet stiffness.

\section{Results of Comparison. The Flexural Rigidity.}

The model as defined above was run for different values of the inertia factor " $f$ " for the same loading case. After several iterations, the resulting value of the factor $f$ was 1 , for the vertical plane. The deflections calculated with the factor $f=1$ fit well the measurements. Using the same value for $f$, the other loading cases were calculated and the results were superposed on the measurements. As Fig. 4 shows, all cases confirmed that the flexural rigidity of the dipole cold mass in its vertical plane can be considered identical to that of the shrinking cylinder, being $142 \mathrm{MNm}^{2}$.

A similar FE model, representing the conditions of the second set up (Fig. 7), allows to determine the horizontal stiffness of 


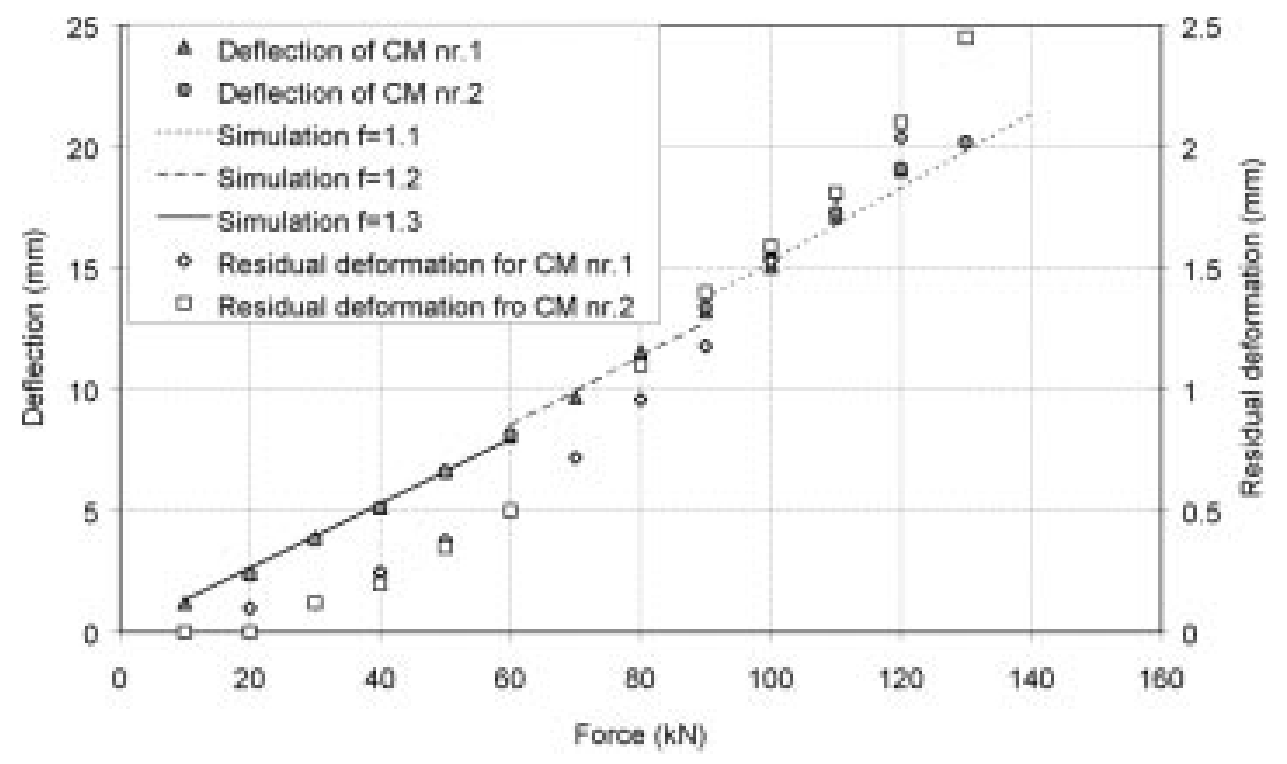

Fig. 8. Variation of the flexural rigidity of the cold mass in its transversal plane, during plastic deformation.

the CM. Varying the estimated magnet stiffness (i.e., the factor " $f$ "), the results were fitted to the deflections measured on a CM rotated 90 degrees with respect to its nominal position. This results in a factor $f=1.3$, meaning that the flexural rigidity of the cold mass in the transversal plane exceeds the rigidity in the vertical plane (as concluded from Fig. 6), by $30 \%$.

\section{Plastic Behavior of the Dipole Cold Mass}

The dipole cold mass curvature in the transversal plane (as shown in Fig. 1) is obtained by placing the magnet on a curved press table and, under press load, welding the two half-cylinders to form a skin around the active part. When the load is released after welding, the magnet loses a nonnegligible fraction of the curvature due to elastic spring back. To compensate the spring back, the press table is shaped to a slightly higher curvature (smaller radius) than that of the CM nominal shape, but several attempts were needed to determine the correct press table shape. From those trials, some cold masses ended up being insufficiently or too strongly curved. These cold masses were re-shaped under the press table. A set-up to "decrease" the curvature of the $\mathrm{CM}$ is shown in Fig. 7. Three cold masses were measured in the same conditions, showing the same behavior. For different values of the applied force, the deflection of the CM extremity is measured, both under load and after unloading. Fig. 8 shows the measured deformations measured on two cold masses, and compares them with simulated deformations under load with " $f$ " $=1.3,1.2$ and 1.1 . For loads up to $50 \mathrm{kN}$, the previ- ously determined value of 1.3 for the stiffness factor " $f$ " in the elastic range is confirmed, but once the plastic range reached, the magnet stiffness decreases.

\section{CONCLUSIONS}

A finite element mechanical model has been used to determine the flexural rigidity of the cold mass via inverse calculation. A sensitivity study of the model shows the necessity of accurate knowledge of the input parameters. Combining different sets of deformation measurements with the finite element results, the rigidity of the cold mass is found equal to the rigidity of the shrinking cylinder in the magnet's vertical plane and $30 \%$ higher in the transverse plane. During the various deformation tests, no cross-talk between the vertical and transverse planes was found.

\section{ACKNOWLEDGMENT}

The authors would like to thank M. Duret, C. Lopez, and the team of the Magnet Assembly Facility at CERN for their help.

\section{REFERENCES}

[1] M. Bajko, F. Savary, and W. Scandale, "Geometry and alignment requirements for the LHC main dipole," in EPAC 2000, Vienna, Austria, 2000.

[2] Technical Specification for the Supply of 1158 Cold Masses of the Superconducting Dipole Magnets for the LHC Collider, LHC Project document nr. LHC-MB-CI-0006, May 2001. 